\section{Characterization of some modern scintillators recommended for use on large fusion facilities in $\gamma$-ray spectroscopy and tomographic
measurements of $\gamma$-emission profiles}

Pawel Sibczynski, Andrzej Broslawski, Aneta Gojska, Vasili Kiptily, Stefan Korolczuk, Roch Kwiatkowski, Slawomir Mianowski, Marek Moszyński, Jacek Rzadkiewicz, Lukasz Swiderski, Adam Szydlowski, Izabella Zychor

\begin{abstract}
LaBr}_{3}: \mathrm{Ce}, \mathrm{CeBr}_{3}$ and GAGG:Ce scintillators were investigated and the determined characteristics were compared with those obtained for the well-known and widely used CsI:Tl and NaI:Tl crystals. All the detectors were of the same size of $10 \times 10 \times 5 \mathrm{~mm}^{3}$. The aim of this test study was to single out scintillation detectors most suitable for $\gamma$-ray spectrometry and $\gamma$-ray emission radial profile measurements in high-temperature plasma experiments. Decay time, energy resolution, non-proportionality and full energy peak detection efficiency were measured for $\gamma$-ray energies up to $1770 \mathrm{keV}$. Due to their good energy resolution, short decay time and high detection efficiency for $\mathrm{MeV}$ gamma rays, $\mathrm{LaBr}_{3}$ : $\mathrm{Ce}$ and $\mathrm{CeBr}_{3}$ scintillators are proposed as the best candidates for use especially under conditions of high count rates, which are expected in the forthcoming DT experiments.
\end{abstract}

Keywords: gamma spectrometry $\bullet$ scintillators $\bullet$ decay time $\bullet$ non-proportionality $\bullet$ energy resolution $\bullet$ detection efficiency • fusion plasma

P. Sibczynski, A. Broslawski, A. Gojska, S. Korolczuk,

R. Kwiatkowski, S. Mianowski, M. Moszyński,

J. Rzadkiewicz, L. Swiderski, A. Szydlowski ${ }^{\bowtie}$,

I. Zychor

National Centre for Nuclear Research (NCBJ),

7 Andrzeja Sołtana Str., 05-400 Otwock/Swierk, Poland,

Tel.: +48 22273 1534, Fax: +48 22779 3481,

E-mail: Adam.Szydlowski@ncbj.gov.pl

V. Kiptily

Culham Centre for Fusion Energy (CCFE),

Culham Science Centre,

OX14 3DB, Abingdon, United Kingdom

Received: 13 May 2016

Accepted: 25 January 2017

\section{Introduction}

The hard X-ray (HXR) and $\gamma$-ray diagnostics of fast ion behaviour in magnetically confined plasmas, used presently at various tokamaks [1-3] and foreseen for the ITER experimental device, provide crucial information on different characteristics of fast electrons, fusion reaction products and other fast primary ions. The energetic ions give rise to the intense $\gamma$-ray emission reacting with either fuel ions or main plasma low-Z impurities, that is, beryllium and carbon $[4,5]$. HXR and $\gamma$-ray intensities, as well as their energy spectra recorded with collimated spectrometers deliver essential data on energy distributions of fast electrons and energetic ions (fusion reaction products, ion-cyclotron resonance frequency (ICRF) - accelerated ions, NBI-injected ions). Also, tomographic reconstruction of the radial profile of $\gamma$-ray emission as is performed on a few tokamaks (JET, TFTR, FTU) with a neutron profile monitor is a direct source of information on the radial distribution of the fast ions. At ITER, the $\gamma$-ray spectrometry will be used for fusion $\alpha$-particle studies needed for the understanding of burning plasma physics, and HXR profile measurements will be performed for the monitoring of runaway electrons, which are undesirable in plasma [6]. Time-resolved $\gamma$-ray emission profile measurements are used to investigate the time evolution of processes taking place in the plasma volume during high temperature discharges. Time-dependent 
spatial distribution of $\gamma$-ray emission sources are measured on JET with two cameras viewing the plasma along horizontal and vertical lines of sight.

The ideal detector for HXR and $\gamma$-ray diagnostics of tokamak plasmas should then be characterized by a fast response to incident radiation. This is especially important for deuterium-tritium (DT) experiments in which high counting rates up to a few $\mathrm{MHz}$ are expected. A fast response of the detectors reduces the dead-time of data acquisition system and minimizes the pile-up effects.

Another important parameter of a $\gamma$-ray spectrometer is its energy resolution, which depends mainly on the detector material. The energy resolution also depends on non-proportionality of the signal output to the incident radiation. As amplitude of a signal is proportional to the detector light output, the term 'non-proportionality of light output' is widely used, often abbreviated just as 'non-proportionality'. In the case of $\gamma$-ray cameras, modernized at JET, it is expected to obtain an energy resolution equal or better than $5 \%$ at $\gamma$-ray energy equal to $1.1 \mathrm{MeV}$ [7].

The next decisive parameter is the detection efficiency of the $\gamma$-radiation detector. Materials with high densities and high atomic numbers are preferable because the detection efficiency depends on these characteristics of the detector material [8]. In addition, the detectors should be resistant to harsh conditions existing in fusion plasma experiments. Indeed, the detectors will operate in a strong magnetic field (up to $5 \mathrm{~T}$ ) and intensive neutron fluxes, as the total neutron yield during ITER DT experiments is expected to be up to $10^{20}$ neutrons per second [9].

Scintillators are typically used in detector systems for HXR and $\gamma$-ray diagnostics, and they are good candidates for DT tokamak experiments [10]. These materials can be produced in relatively large size pieces up to several inches and do not require cooling for operation that is an advantage in comparison with semiconductor materials; also, because of the space limitation for detector installation (e.g., in $\gamma$-ray and neutron cameras in tokamak).

All the scintillators used in this work (see Table 1 of the selected scintillators) were of the same size of $10 \times 10 \times 5 \mathrm{~mm}^{3}$ in order to perform comparative tests for detectors considered for the tokamak $\gamma$-ray diagnostic. Since decay time, energy resolution and non-proportionality do not strongly depend on a crystal size, the presented results are helpful for further selection of high performance scintillation detectors for upgraded $\gamma$-ray diagnostics for JET DT campaigns [11, 12].

It is interesting to note that in contrast to $\mathrm{LaBr}_{3}: \mathrm{Ce}, \mathrm{NaI}: \mathrm{Tl}$ and $\mathrm{CsI}: \mathrm{Tl}$ detectors that were already used in JET experiments in HXR and $\gamma$-ray diagnostic systems $[13,14], \mathrm{CeBr}_{3}$ and GAGG:Ce (gadolinium aluminium gallium garnet - structural notation of $\mathrm{Gd}_{3} \mathrm{Al}_{2} \mathrm{Ga}_{3} \mathrm{O}_{12}: 1 \% \mathrm{Ce}$ ) scintillators have not been considered for use in plasma experiments so far. Since capabilities of scintillation detectors depend on setup geometry, crystal size, photomultiplier types, etc., the described measurements were made under the same experimental conditions.

The following quantities for each tested scintillator were measured: decay time of a light pulse, energy resolution, non-proportionality and full energy peak detection efficiency of $\gamma$-rays. Based on the obtained results, a scintillating material, suitable for the HXR and $\gamma$-ray tokamak diagnostics and appropriate for high count rate measurements, is recommended.

\section{Experimental details}

A comparative study of all tested scintillator samples was performed using the experimental setup shown in Fig. 1.

All the $10 \times 10 \times 5 \mathrm{~mm}^{3}$ scintillators have a cuboid shape. Because of a hygroscopic nature of NaI:Tl, $\mathrm{LaBr}_{3}: \mathrm{Ce}$ and $\mathrm{CeBr}_{3}$ materials, these crystals were assembled by manufacturers in an aluminium can with a glass window.

Each scintillator was coupled to a photomultiplier (PMT) with a silicone grease to improve the optical contact with a photodetector window. Measurements of a specific scintillator parameter were done with a photomultiplier chosen for an optimal performance. Two types of photomultipliers were used: Hamamatsu R6231-100 and Hamamatsu R5320.

The scintillation decay time was measured using a Hamamatsu R5320 photomultiplier because of its fast response characteristics. In order to determine the response time, the anode pulses were recorded and averaged by a Tektronix digital oscilloscope.

A scintillator energy resolution, non-proportionality and full energy peak detection efficiency as a function of $\gamma$-ray energy were measured with a Hamamatsu R6231-100 PMT. The anode signal from the photomultiplier was amplified by a Canberra 2005 preamplifier and shaped by an Ortec 672 spectroscopy amplifier. Energy spectra were recorded with a Tukan 8K USB multi-channel analyzer [15].

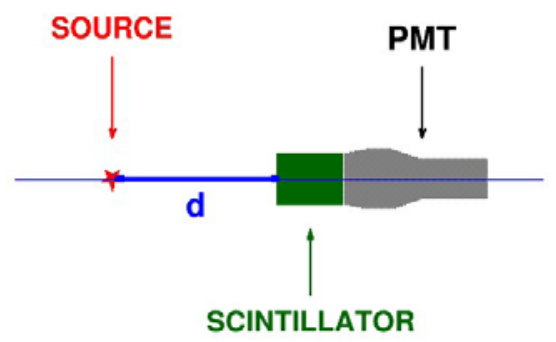

Fig. 1. Schematic of the experimental setup used in the described measurements, $d$ - distance between the $\gamma$-ray source position and the front surface of the scintillator.

Table 1. Density and effective atomic number $Z_{\text {eff }}$ of the tested scintillator materials with information about manufacturer

\begin{tabular}{lccccc}
\hline \multicolumn{1}{c}{ Scintillator } & CsI:Tl & $\mathrm{NaI}: \mathrm{Tl}$ & $\mathrm{LaBr}_{3}: \mathrm{Ce}$ & $\mathrm{CeBr}_{3}$ & $\mathrm{GAGG:Ce}$ \\
\hline Density $\left[\mathrm{g} / \mathrm{cm}^{3}\right]$ & 4.51 & 3.67 & 5.06 & 5.18 & 6.63 \\
$\mathrm{Z}_{\text {eff }}$ & 54.0 & 49.7 & 45.2 & 45.9 & 50.5 \\
Manufacturer & Amcrys-H & Scionix & St-Gobain & Scionix & Furukawa \\
\hline
\end{tabular}


$\mathrm{X}$ - and $\gamma$-ray sources with energies from $16.6 \mathrm{keV}$ up to $1770 \mathrm{keV}$ were used in these test measurements.

We assessed that in general, the statistical error (square root of count number) makes the main source of errors in determining the integral and full width at half maximum (FWHM) of the recorded peak. The peak position was estimated with an accuracy of $\pm 1.5 \%$. An accuracy of the geometry setting (the distance between the $\gamma$-source position and the scintillator top surface) was evaluated to be $\pm 2 \mathrm{~mm}$. A source activity was estimated with an accuracy of $\pm 3.5 \%$.

\section{Results}

\section{Decay time}

Relaxation of the light emission produced in scintillators by incident photon is characterized by a decay time parameter. Typically, the scintillation decay time varies between nanoseconds and microseconds. For some scintillators, more than one decay modes are observed, such as for CsI:Tl [16] and GAGG:Ce [12]. The pulse shapes obtained from the tested scintillators are shown in Fig. 2, scaled at the time stamp of zero.

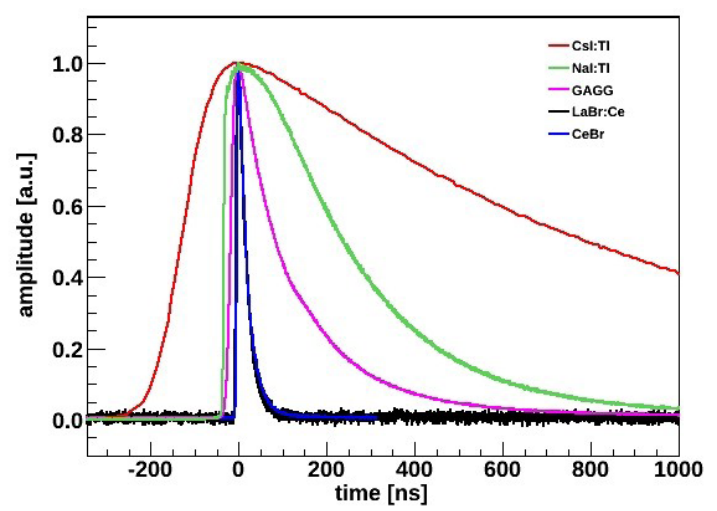

Fig. 2. Pulse shapes obtained from the tested scintillators.

Table 2. Decay times of the light emission from the investigated crystals. Two components, faster and slower, were determined for GAGG:Ce and CsI:Tl and their relative intensities are given in brackets

\begin{tabular}{lcc}
\hline \multirow{2}{*}{ Crystal } & \multicolumn{2}{c}{ Decay time [ns] } \\
\cline { 2 - 3 } & Fast component & Slow component(s) \\
\hline CsI:Tl & $780 \pm 50(46 \%)$ & $3100 \pm 300(29 \%)$ \\
$\mathrm{NaI}: \mathrm{Tl}$ & $230 \pm 10$ & - \\
$\mathrm{LaBr}_{3}: \mathrm{Ce}$ & $18 \pm 2$ & - \\
$\mathrm{CeBr}$ & $19 \pm 2$ & - \\
GAGG:Ce & $140 \pm 5(67 \%)$ & $570 \pm 15(33 \%)$ \\
\hline
\end{tabular}

Each crystal was irradiated with the same ${ }^{137} \mathrm{Cs}$ source. Measured decay times for each tested scintillator are presented in Table 2 .

The fastest response is observed for $\mathrm{LaBr}_{3}: \mathrm{Ce}$ and $\mathrm{CeBr}_{3}$ scintillators. This fact promotes both materials as good candidates for tokamak plasma experiments in which high fluxes of $\gamma$-rays are expected. The responses of GAGG:Ce and NaI:Tl are one order of magnitude slower, however their decay times are not excessively long. Nevertheless, detectors based on CsI:Tl crystal are less useful for high count rate measurements in tokamak plasma diagnostics because its two intense components of 3100 ns and 13600 ns are enormously slow.

\section{Energy resolution}

The energy resolution was measured for $\mathrm{X}$ - and $\gamma$-rays with energies between $16.6 \mathrm{keV}$ and $1770 \mathrm{keV}$. The activities of the used $\gamma$-ray sources were from tens to hundreds kBq. Figure 3 shows, as an example, the energy spectrum of $661.7 \mathrm{keV} \gamma$-ray line emitted from a ${ }^{137} \mathrm{Cs}$ source measured with the $\mathrm{LaBr}_{3}: \mathrm{Ce}$ scintillator. The full energy (total absorption) peak is recorded at energy $661.7 \mathrm{keV}$. In lower energy range, one can observe a Compton continuum, with a Compton edge equal to $477.3 \mathrm{keV}$. As $\gamma$-ray quanta may also be scattered in a backward direction, the backscatter peak is also noticeable at $184.3 \mathrm{keV}$. One can also notice an absorption peak of $32 \mathrm{keV}$ line being a result of KX-ray radiation emitted by the ${ }^{137} \mathrm{Cs}$ source.

In Table 3, a comparison of the energy resolution of all the tested scintillators for different $\gamma$-ray energies as measured with a Hamamatsu R6231-100 PMT is presented. The energy resolution (FWHM) was determined by fitting the total absorption peak with a Gaussian function. The energy resolution dependence on the $\gamma$-ray energy is also shown in Fig. 4 for all the tested scintillators.

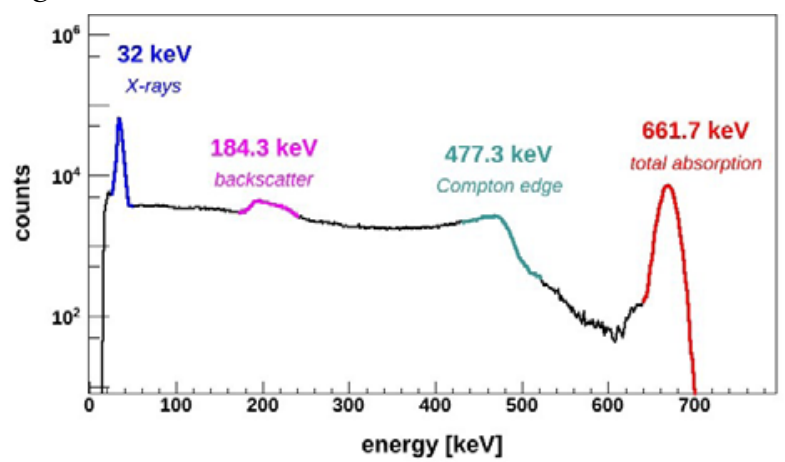

Fig. 3. Energy spectrum of $661.7 \mathrm{keV} \gamma$-ray line from a ${ }^{137} \mathrm{Cs}$ source measured with the $\mathrm{LaBr}_{3}$ :Ce scintillator.

Table 3. Energy resolution (FWHM) measured with a Hamamatsu R6231-100 PMT

\begin{tabular}{lcccc}
\hline \multirow{2}{*}{ Crystal } & \multicolumn{4}{c}{ FWHM [\%] } \\
\cline { 2 - 5 } & $59.5 \mathrm{keV}\left({ }^{241} \mathrm{Am}\right)$ & $661.7 \mathrm{keV}\left({ }^{137} \mathrm{Cs}\right)$ & $1115.5 \mathrm{keV}\left({ }^{65} \mathrm{Zn}\right)$ & $1770 \mathrm{keV}\left({ }^{207} \mathrm{Bi}\right)$ \\
\hline CsI:Tl & $12.5 \pm 0.3$ & $5.7 \pm 0.2$ & $4.9 \pm 0.1$ & $3.8 \pm 0.1$ \\
NaI:Tl & $9.4 \pm 0.2$ & $6.8 \pm 0.2$ & $5.9 \pm 0.2$ & $4.6 \pm 0.1$ \\
LaBr $_{3}$ :Ce & $10.8 \pm 0.3$ & $2.9 \pm 0.1$ & $2.4 \pm 0.1$ & $2.1 \pm 0.1$ \\
CeBr $_{3}$ & $15.7 \pm 0.4$ & $4.4 \pm 0.1$ & $3.4 \pm 0.1$ & $2.9 \pm 0.1$ \\
GAGG:Ce & $23.9 \pm 1.0$ & $6.6 \pm 0.2$ & $3.7 \pm 0.2$ & $3.7 \pm 0.1$ \\
\hline
\end{tabular}




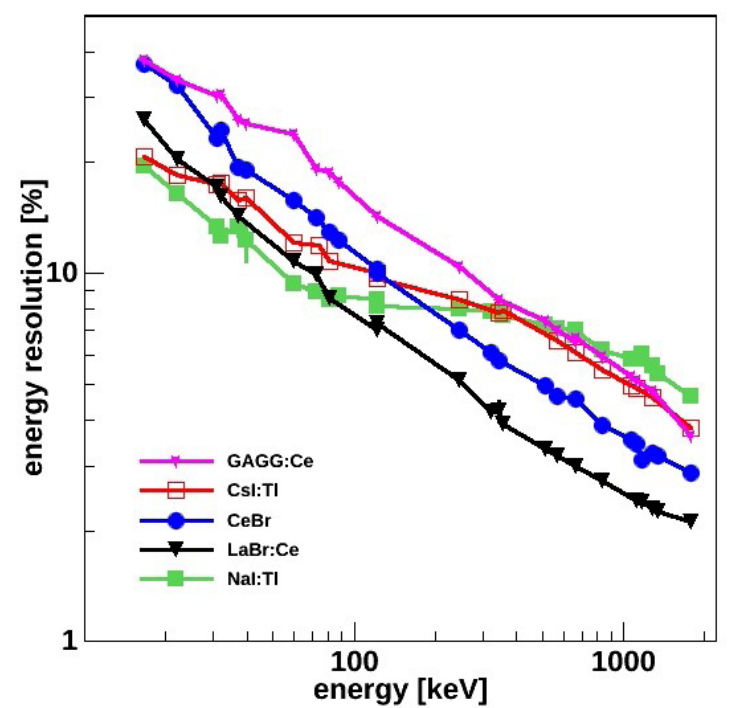

Fig. 4. Energy resolution (FWHM) measured for different $\gamma$-ray lines with energies between $16.6 \mathrm{keV}$ and $1770 \mathrm{keV}$. If not shown, error bars are within a point size.

The $\mathrm{LaBr}_{3}$ :Ce scintillator characterized by an energy resolution equal to $2.9 \%$ at $661.7 \mathrm{keV}$ and $2.1 \%$ at $1770 \mathrm{keV}$ offers the best performance for experimental analysis of gamma rays of energy above $100 \mathrm{keV}$. This is mostly due to a linear response to gamma rays of energy from a quite broad range. This feature will be pointed out below. In the low energy range, CsI:Tl and NaI:Tl show similar performance to $\mathrm{LaBr}_{3}: \mathrm{Ce}$. This is also related to non-proportionality, discussed in the next subsection.

\section{Non-proportionality of light output}

A deviation from a linear response to $\gamma$-ray energy has a noticeable impact on the measured energy resolution of a scintillator [17]. To assess this contribution, the amplitude of signal registered at a specific $\gamma$-ray energy were divided by the amplitude of the $661.7 \mathrm{keV} \gamma$-ray absorption peak and presented as a function of $\gamma$-ray energy (Fig. 5).

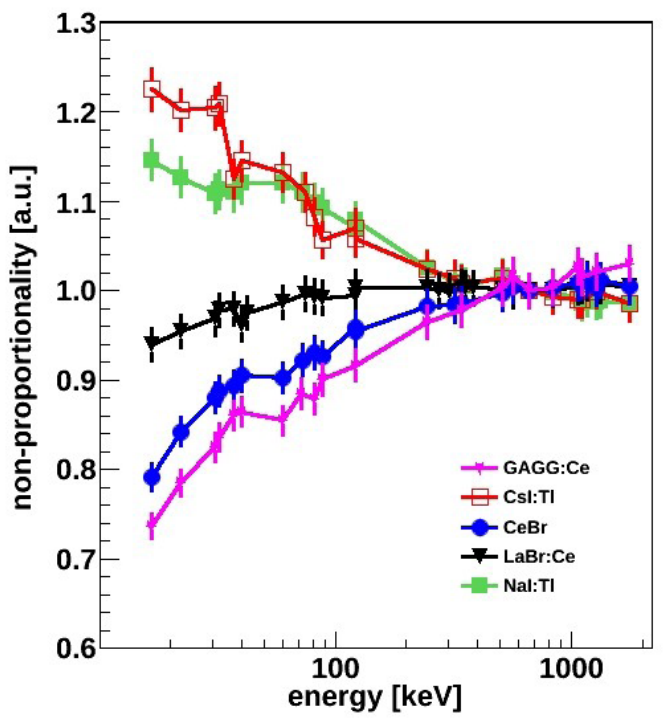

Fig. 5. Non-proportionality of light output as a function of $\gamma$-ray energy relative to the $661.7 \mathrm{keV} \gamma$-peak.
The $\mathrm{LaBr}_{3}$ :Ce shows nearly proportional response to gamma rays at all energies up to $1770 \mathrm{keV}$. $\mathrm{The} \mathrm{CeBr}_{3}$ and GAGG:Ce present a noticeable worse linearity that results in a poorer energy resolution than observed for $\mathrm{LaBr}_{3}$ :Ce. Thallium doped alkali iodides, NaI:Tl and CsI:Tl, demonstrate a quite different response to $\gamma$ quanta. A non-proportionality reaches a constant value for $\gamma$-ray energy above $500 \mathrm{keV}$, increases with decreasing energy deposition below $200 \mathrm{keV}$ and is relatively flat, about $30 \mathrm{keV}$. Such a response results in a substantial improvement of the energy resolution at low energy range, where the measured values are close to those obtained for $\mathrm{LaBr}_{3}: \mathrm{Ce}$.

\section{Full energy peak detection efficiency}

The full energy peak detection efficiency was determined for $\gamma$-ray sources with energies from $22.1 \mathrm{keV}$ up to $1408 \mathrm{keV}$. The measurements were performed for identical setup, with a radioactive source placed at a distance $d=54 \mathrm{~mm}$ from the top surface of the scintillator, see Fig. 1. The full energy peak detection efficiency $\gamma$ is determined by a number $N$ of net counts in the full energy peak area, a source activity $A$, a measurement time $T$, a detection solid angle $\Omega$ and a branching ratio $B$ of the reaction resulting in $\gamma$-ray emission, according to Eq. (2):

$$
\varepsilon=\frac{N}{A \times T \times \Omega \times B}
$$

The determined detection efficiency values are shown in Fig. 6 as a function of $\gamma$-ray energy. To highlight the differences in the full energy peak detection efficiency values for higher $\gamma$-ray energies, the dependence is also shown in the inset in a double logarithmic scale.

The GAGG:Ce sample has the highest detection efficiency among all the tested scintillator crystals. At the highest used $\gamma$-ray energy equal to $1408 \mathrm{keV}$, the GAGG:Ce is much more efficient than other scintillators of the same size. This is due to the

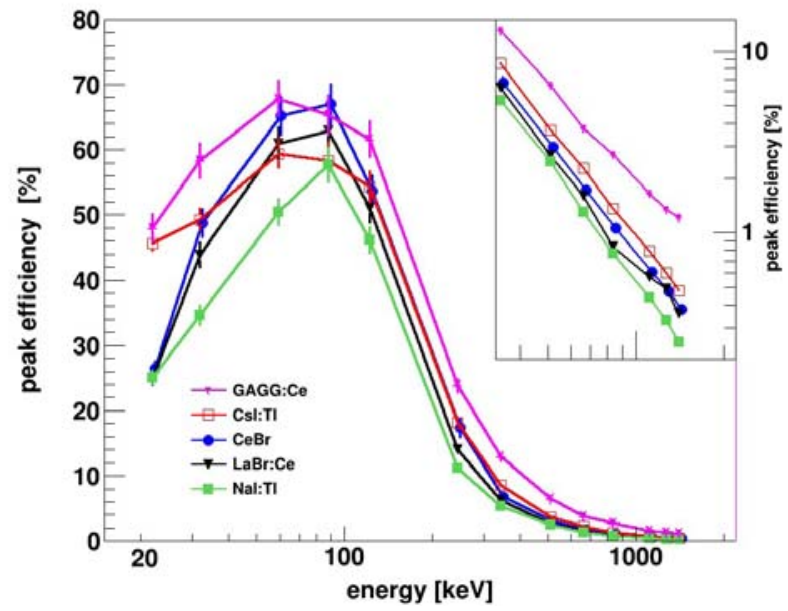

Fig. 6. Full energy peak detection efficiency measured for $\gamma$-rays with energies from $22.1 \mathrm{keV}$ to $1408 \mathrm{keV}$. In the inset, the dependence for higher energies is shown in a double logarithmic scale. Lines are a guide for the eye. 
higher density of GAGG:Ce scintillator in comparison with other tested materials.

A drop in the detection efficiency observed for all the tested scintillators in the low energy range is due to the following reasons:

1. An attenuation of X-rays and low energy $\gamma$-rays by wrapping and encapsulation materials.

2. A steep drop of the absorption probability of $\mathrm{X}$-rays for energies lower than K-shell electron binding energy (K-shell absorption edge), depending on the scintillation material composition (effective atomic number).

3. Relatively high probability of KX-ray escape from small crystals. KX-rays interact with the scintillator material solely via photo absorption process. At energies $>300 \mathrm{keV}$, the width of the full energy peak is so large, that it merges with the KX-ray escape peak and in consequence KX-ray escape events are confined in the integral of absorbed quanta. For lower energies, KX-ray escape events form a separate peak, in turn reducing a large number of counts from the full energy peak. Using crystals with larger volumes would reduce this effect.

\section{Conclusions and outlook}

Scintillation detectors $\mathrm{LaBr}_{3}$ : $\mathrm{Ce}$ and $\mathrm{CeBr}_{3}$, GAGG:Ce, CsI:Tl and NaI:Tl with the same size of $10 \times 10 \times 5 \mathrm{~mm}^{3}$ have been characterized. The crystals were irradiated by X-and $\gamma$-rays with energies from $16.6 \mathrm{keV}$ up to $1770 \mathrm{keV}$. Measurements were performed in the same experimental conditions with optimally matched photomultipliers. Since decay time, energy resolution and non-proportionality do not strongly depend on a crystal size, presented results allow us to draw general conclusions on possible use of the investigated scintillators as HXR and $\gamma$-ray diagnostic tools dedicated for high count rate measurements of $\gamma$-rays emitted from tokamak plasmas.

The measured properties of all the tested scintillators are summarized as follows:

1. The fastest decay time ( $\sim 20 \mathrm{~ns})$ was confirmed for $\mathrm{LaBr}_{3}$ : $\mathrm{Ce}$ and $\mathrm{CeBr}_{3}$, which favours these two materials used in experiments with high count rates in high-performance tokamak plasma conditions. Measurements with a $\mathrm{LaCl}_{3}$ :Ce scintillator, which is characterized by a decay time equal to $28 \mathrm{~ns}$, were already performed at high count rates $\sim 2 \mathrm{MHz}$ [18].

2. In the energy range above $100 \mathrm{keV}, \mathrm{LaBr}_{3}: \mathrm{Ce}$ has the best energy resolution due to its almost perfect linearity of $\gamma$-ray response and its highest light yield among the investigated crystals. At energies below $100 \mathrm{keV}$, CsI:Tl and NaI:Tl show comparable energy resolution.

3. Although, the CsI:Tl is characterized by a reasonable high full energy peak detection efficiency of $(2.2 \pm 0.2) \%$ at $\gamma$-ray energy of $661.7 \mathrm{keV}$, a long response time limits its detection capability in the case of high count rate measurements.

4. The NaI:Tl scintillator is much faster than CsI:Tl. It is a relatively low-cost material, however, it shows the worst measured energy resolution, particularly in high energy range, as well as the lowest full energy peak detection efficiency.

5. The GAGG:Ce has the highest detection efficiency among all the tested scintillator crystals and an energy resolution comparable to that obtained with CsI:Tl. However, as a consequence of the highest non-proportionality, the poorest energy resolution in the low energy range was observed. The GAGG:Ce shows a fast component of decay time equal to $140 \mathrm{~ns}$ and is characterized by the highest full energy peak detection efficiency in the investigated $\gamma$-ray energy range.

Initially, our team considered using the GAGG:Ce scintillator [12] in plasma experiments as it has the highest full energy peak detection efficiency in the gamma energy range between 1.0 and $10 \mathrm{MeV}$, see Fig. 6. Also, it is characterized by relatively short scintillation decay time $(\sim 140 \mathrm{~ns})$. These two parameters could recommend this material for plasma experiment. However, GAGG:Ce scintillators contain elements, particularly oxygen, which have a relatively high cross section for inelastic scattering of fast neutrons and for ${ }^{16} \mathrm{O}(\mathrm{n}, \mathrm{p}){ }^{16} \mathrm{~N}$ nuclear reactions. These reactions produce undesirable additional gamma background.

Detection efficiency strongly depends on the scintillator size. So, it is important to make a detailed study with crystals of different sizes [11]. Measurements with sources emitting a few lines of $\gamma$-rays of energy in a $\mathrm{MeV}$ range are in progress [19].

Being aware of the fact that detectors for HXR and $\gamma$-ray tokamak plasma diagnostics will be used in a strong magnetic field which can affect the photomultiplier performance, semiconductor photodiodes (Silicon Photo Multiplier, Multi-Pixel Photon Counter (MPPC), Avalanche PhotoDiode) have also been considered as an option for experiments performed in a strong magnetic field. The conclusions drawn in this work for $\mathrm{LaBr}_{3}$ :Ce and $\mathrm{CeBr}_{3}$ crystals coupled to PMT are also valid for scintillators used with MPPC photodetectors having a gain of $10^{5}$, which is comparable to that obtained with classic PMTs. This approach is now investigated for using in tokamak plasma experiments [20] in which high count rates are foreseen. In addition, a possibility to use a PiN diode in $\gamma$-ray spectrometry instead of a PMT, was investigated at the NCBJ. However, such a solution seems to be less feasible for $\gamma$-ray diagnostics intended for experiments in which high count rates will occur, because the scintillator light pulses are not amplified by a PiN diode significantly enough to cause that electronic noises exert a significant impact on the measured energy resolution.

Summarizing, the experiments in which high count rates are expected and the registered gamma rays will have energies above $100 \mathrm{keV}$, the detector systems based on $\mathrm{LaBr}_{3}: \mathrm{Ce}$ and $\mathrm{CeBr}_{3}$ scintillators are the best candidates. During the future extensive DT campaign at JET, in which high neutron and $\gamma$-ray fluxes are expected, a performance of detectors, based on $\mathrm{CeBr}_{3}$ and $\mathrm{LaBr}_{3}$ :Ce scintillators will be investigated. The obtained results will then be used to conclude about a possible use of such detectors at ITER for $\gamma$-ray diagnostics. 
For lower count rates, the well-known and widely used CsI:Tl and NaI:Tl scintillators are still a reasonable option.

Acknowledgments. This scientific work was partly supported by the Polish Ministry of Science and Higher Education within the framework of scientific financial resources in the years 2015-2017, allocated for the realization of the international co-financed project.

\section{References}

1. Cecil, F. E., \& Medley, S. S. (1988). Gamma ray measurements during deuterium and $3 \mathrm{He}$ discharges on TFTR. Nucl. Instrum. Methods Phys. Res. Sect. A-Accel. Spectrom. Dect. Assoc. Equip., 271(3), 628-635. DOI: 10.1016/0168-9002(88)90333-6.

2. Sadler, G. I., Convoy, S. W., Jarvis, O. N., Belle van, P., Adams, J. M., \& Hone, M. A. (1990). Investigations of fast particle behavior in JET plasmas using nuclear techniques. Fusion Technol., 18, 556.

3. Kondoh, T., Kusama, Y., Kimura, H., Saigusa, M., Fujii, T., Moriyama, S., Nemoto, M., Tobita, K., Morioka, A., Nagashima, K., \& Nishitani, T. (1997). Investigation of interaction between $\mathrm{MeV}$-ions and first wall from neutron and $\gamma$-ray measurements in JT-60U. J. Nucl. Mater., 564, 241-243. DOI: 10.1016/ S0022-3115(97)80100-6.

4. Esposito, B., Martin Solis, R., Belle van, P., Jarvis, O. N., Marcus, F. B., Sadler, G., Sanchez, R., Fischer, B., Froissard, P., Adams, J. M., Cecil, E., \& Watkins, N. (1996). Runaway electron measurements in the JET tokamak. Plasma Phys. Control Fusion, 38, 2035-2049. http://dx.doi.org/10.1088/0741$3335 / 38 / 12 / 001$

5. Kiptily, V. G., Cecil, F. E., \& Medley, S. S. (2006). Gamma ray diagnostics of high temperature magnetically confined fusion plasmas. Plasma Phys. Control. Fusion, 48, R59-R82. http://dx.doi. org/10.1088/0741-3335/48/8/R01.

6. Chugunov, I. N., Shevelev, A. E., Gin, D. B., Kiptily, V. G., Gorini, G., Nocente, M., Tardocchi, M., Doinikov, D. N., Naidenov, V. O., \& Khilkevitch, E. M. (2011) Development of gamma-ray diagnostics for ITER. Nucl. Fusion, 51, 083010. http://dx.doi. org/10.1088/0029-5515/51/8/083010.

7. JET. (2014). JET Enhancements Programme WPJET4. (internal document).

8. Knoll, G. (2010). Radiation detection and measurement (4th ed.). USA: John Wiley \& Sons, Inc.

9. Sasao, M., Bertalot, L., Ishikawa, M., \& Popovichev, S. (2010). Strategy for the absolute neutron emission measurement on ITER. Rev. Sci. Instrum., 81, 10D329. http://dx.doi.org/10.1063/1.3491049.

10. Kiptily, V. G., Popovichev, S., Sharapov, S. E., Bertalot, L., Cecil, F. E., Conroy, S., \& Mantsinen, M. J. (2003). Gamma-diagnostics of alpha-particles in ${ }^{4} \mathrm{He}$ and D-T plasmas. Rev. Sci. Instrum., 74, 1753. http:// dx.doi.org/10.1063/1.1534922.

11. Swiderski, L., Gojska, A., Grodzicka, M., Korolczuk, S., Mianowski, S., Moszynski, M., Rzadkiewicz, J., Sibczynski, P., Syntfeld-Kazuch, A., Szawlowski, M., Szczesniak, T., Szewinski, J., Szydlowski, A., \& Zychor, I. (2015). Scintillators for high temperature plasma diagnostics. In Proceedings of the 1st EPS
Conference on Plasma Diagnostics. Available from http://pos.sissa.it/archive/conferences/240/162/ ECPD2015 162.pdf.

12. Sibczyński, P., Iwanowska-Hanke, J., Moszyński, M., Swiderski, L., Szawłowski, M., Grodzicka, M., Szczęśniak, T., Kamada, K., \& Yoshikawa, A. (2015). Characterization of GAGG:Ce scintillators with various Al-to-Ga ratio. Nucl. Instrum. Methods Phys. Res. Sect. A-Accel. Spectrom. Dect. Assoc. Equip., 772, 112-117. http://dx.doi.org/10.1016/j.nima.2014.10.041.

13. Nocente, M., Tardocchi, M., Chugunov, I., Pereira, R. C., Edlington, T., Fernandes, A. M., Gin, D., Grosso, G., Kiptily, V., Murari, A., Neto, A., Perelli Cippo, E., Pietropaolo, A., Proverbio, I., Shevelev, A., Sousa, J., Syme, B., Gorini, G., \& JET-EFDA Contributors. (2010). Energy resolution of gamma-ray spectroscopy of JET plasmas with a $\mathrm{LaBr}_{3}$ scintillator detector and digital data acquisition. Rev. Sci. Instrum., 81 10D321. http://dx.doi.org/10.1063/1.3501386.

14. Kiptily, V. G., Adams, J. M., Bertalot, L., Murari, A., Sharapov, S. E., Yavorskij, V., Alper, B., Barnsley, R., de Vries, P., Gowers, C., Eriksson, L. G., Lomas, P. J., Mantsinen, M. J., Meigs, A., Noterdaeme, J. M., \& Orsitto, F. P. (2005). Gamma-ray imaging of D and $4 \mathrm{He}$ ions accelerated by ion-cyclotron-resonance heating in JET plasmas. Nucl. Fusion, 45, L21-L25.

15. Guzik, Z., Borsuk, S., Traczyk, K., \& Płomiński, M (2006). TUKAN - an 8K pulse height analyzer and multi-channel scaler with a PCI or a USB interface. IEEE Trans. Nucl. Sci., 53, 231-235. http://dx.doi org/10.1109/TNS.2006.869819.

16. Syntfeld-Każuch, A., Moszynski, M., Swiderski, L., Klamra, W., \& Nassalski, A. (2008). Light pulse shape dependence on $\gamma$-ray energy in CsI(Tl). IEEE Trans. Nucl. Sci., 55, 1246-1250. http://dx.doi.org/10.1109/ TNS.2008.922805.

17. Swiderski, L., Marcinkowski, R., Szawlowski, M., Moszynski, M., Czarnacki, W., Syntfeld-Kazuch, A., Szczesniak, T., Pausch, G., Plettner, C., \& Roemer, K. (2012). Non-proportionality of electron response and energy resolution of Compton electrons in scintillators. IEEE Trans. Nucl. Sci., 59, 222-229. http:// dx.doi.org/10.1109/TNS.2011.2175407.

18. Korolczuk, S., Mianowski, S., Rzadkiewicz, J., Sibczynski, P., Swiderski, L., \& Zychor, I. (2016). Digital acquisition in high count rate gamma ray spectrometry. IEEE Trans. Nucl. Sci., 99, PP 1-1. http://dx.doi. org/10.1109/TNS.2016.2567455.

19. Zychor, I., Boltruczyk, G., Burakowska, A., Craciunescu, T., Fernandes, A., Figueiredo, J., Giacomelli, L., Gorini, G., Gierlik, M., Gosk, M., Grodzicka, M., Iwanowska-Hanke, J., Kaveney, G., Kiptily, V., Korolczuk, S., Kwiatkowski, R., Mianowski, S., Moszynski, M., Murari, A., Nocente, M., Pereira, R. C., Perseo, V., Rigamonti, D., Rzadkiewicz, J., Sibczynski, P., Santos, B., Soare, S., Syntfeld-Kazuch, A., Swiderski, L., Szawlowski, M., Szczesniak, T., Szewinski, J., Szydlowski, A., Tardocchi, M., Urban, A., Zoita, V. L., \& JET Contributors. (2016). High performance detectors for upgraded gamma ray diagnostics for JET DT campaigns. Phys. Scripta, 91, 064003.

20. Boltruczyk, G., Gosk, M., Mianowski, S., Szawlowski, M., \& Zychor, I. (2015). Temperature compensation device for MPPC in plasma diagnostics. In International Conference on Research and Applications of Plasmas PLASMA2015, September 2015, Warsaw, Poland. 